\author{
EVS26 \\ Los Angeles, California, May 6-9, 2012
}

\title{
Neighbourhood Electric Vehicles in Mature Suburbs
}

\author{
David Magarian, Walter Seimbab \\ South Bay Cities Council of Grovernments. \\ david.magarian@sbesc.com \\ ws@siembab.com \\ jacki@southbaycities.org
}

\begin{abstract}
This is a Report on the first phase of a demonstration of Neighborhood Electric Vehicles (NEVs) in the South Bay Subregion of Los Angeles County. The project is sponsored by the South Bay Cities Council of Governments (SBCCOG) and funded by the South Coast Air Quality Management District (AQMD). Active use of the first demonstration phase began May 1, 2010 and ran for 18 months ending October $31,2011$.
\end{abstract}

This Report, based on the 18 months of data collection and analysis, aims to identify the positive role NEVs can play in addressing the following issues:

- Reducing green house gas emissions, criteria air pollutants, and consumption of fossil fuels by passenger vehicles and light trucks.

- Informing government plans and policies currently being formulated, including the 2012 Regional Transportation Plan, California Energy Commission's initiative for electric vehicle readiness, and the
California Air Resources Board AB 118

vehicle voucher program.

- Implementing the Sustainable South Bay Strategy with its mobility initiative based on transitioning the gasoline fueled passenger vehicle fleet to some form of electric vehicle.

Because this study is extremely data rich, a very detailed and scientific analysis has been completed for a somewhat small sample size of 29 participating households. From these findings it is clear that significant GHG and criteria air pollutant reductions could be achieved from wide spread use of NEVs for suburban residential driving. This research has also identified numerous market barriers that prevent wide spread adoption of NEVs as well as strategies to overcome market barriers such as production quality, speed limitations, and NEV prices. 


\section{Context - The Sustainable South Bay Strategy}

The South Bay is a mature, built-out suburban area, much like many other places in Southern California. Despite having pockets of residential density among the highest in Los Angeles County, the South Bay sub-region is transit-poor in terms of both bus services and rail infrastructure. If the fifteen incorporated cities of the South Bay were a single city, it would have the population of Portland, Oregon but with about $50 \%$ more residential density and without Portland's transit infrastructure and dominant downtown.

The Sustainable South Bay Strategy is based on the results of a research program that was designed to identify land use and transportation initiatives that would reduce GHG emissions, criteria pollutants and gasoline consumption by building on existing strengths of South Bay cities.

The Board of Directors of the South Bay Cities Council of Governments (SBCCOG) adopted the Sustainable South Bay Strategy (SSBS) in October, 2010 as the basis for the sub-region's contribution to the regional Sustainable Communities Strategy (SCS) and as a guide to land use planning and transportation policy in cities interested in becoming more sustainable.

The Local Use Vehicle (LUV) Demonstration Project provides "proof of concept" of one aspect of the mobility component of the SSBS. SSBS implementation requires much less public sector investment, land use change and social behavioral change than the transit-density strategy typically used to meet SB 375 goals elsewhere in the region. That is possible because the existing development pattern features many horizontal mixed-use neighborhoods where the SBCCOG studies have shown that most trip destinations are within 3 radial miles of home.

The primary land use strategy involves gradually re-organizing low density destinations -especially commercial strips along major arterials -- into compact, higher density centers in the middle of every neighborhood (for example, at the intersections of major arterials). The lowdensity commercial strips can be transitioned into new housing, built at densities compatible with the existing adjacent neighborhoods, rather than at the much higher densities needed to make public transit service more economically feasible (funding to improve transit infrastructure in the South Bay is not expected for at least 20 years). These land use changes should dramatically encourage walking and cycling as mode choices as there will be compact commercial destinations within one-half mile of every home and a regular pattern of similar centers every mile in each direction.

There is more to it of course, but those are the land use basics. This strategy is referred to as "neighborhood oriented development" (NOD as distinguished from transit oriented development or TOD).

While NOD is a long-term strategy for improving proximity between residential origins and the variety of regular destinations, the strategy for reducing the negative impacts of mobility within the existing pattern of destinations can be implemented in the short-term. That transportation strategy is based on transforming the private passenger vehicle fleet from predominantly gasoline-fueled to predominantly plug-in electric (PEV) or some future alternative fuel such as hydrogen fuel cells. There are currently two types of PEVs: 1) Plug-in hybrids (PHEV) like the Chevrolet Volt that are mostly electric but still require small amounts of gasoline; and 2) battery electric vehicles (BEV) that run $100 \%$ on batteries and produce zero tailpipe emissions. BEVs are range-limited; however this constraint is actually a feature compatible with the existing and future development pattern of mature suburbs like the South Bay.

The most significant strengths of this fleettransition strategy are that household mobility will remain anchored in the door-to-door, on-demand service, which minimizes the need for significant changes in travel behavior; and that the primary source of investment will be private households which minimizes the required level of public sector investment.

Although there are segments of the BEV market that will be much lower cost than today's gasoline fueled vehicles, a safety net will be required for those who cannot afford to purchase a vehicle. PEV purchase vouchers, lease-purchase programs, and neighborhood car sharing are among the options. The safety net will also include 
neighborhood-based vanpools, jitneys, and ride sharing - all part of SSBS implementation.

Transit will play a supportive role as it does today, but the SBCCOG expects that will happen through a variety of innovative services that will rely on a regular pattern of compact commercial destinations rather than high levels of residential density. Small-vehicle, short hop circulators and demand-responsive services are more likely to be effective for the many local trips; rapid long-haul lines with the traditional 40 to 57 seat buses are expected to connect the South Bay to regional transit centers and light rail stations more effectively in the future.

The near-term challenge is to begin the transition of the almost 600,000 private passenger vehicles in the South Bay to some form of PEV. The SBCCOG working assumption is that the first vehicle in most households will need to be capable of long distance travel. Therefore, about 350,000 primary vehicles will need to be gradually replaced by the unlimited range plug-in hybrid vehicles (PHEV) beginning to enter the market. Transition of the approximately 250,000 second and third vehicles per household to rangelimited battery electric vehicles (BEVs) can begin immediately.

A number of BEVs are currently on the market with more expected each year. BEVs can operate at freeway speeds. Vehicles vary in their range from 70 - 130 miles. Sorter range runabout $\mathrm{BEVs}$ and long range $\mathrm{BEVs}$ are expected to arrive on the market in 2013.

The BEV niche currently with the most number of model options is the Neighborhood Electric Vehicle (NEV). NEVs have a maximum speed of 25 miles per hour, a range of about 25 miles between charges, and are legal only on mixedflow streets with speed limits of $35 \mathrm{MPH}$ or less. They can also be legally driven on streets with speed limits faster than $35 \mathrm{MPH}$ in a specially designated lane, similar to a Class 2 bike lane.

In summary, the short distances associated with long-term sustainability match the limited range of BEVs. This harmonious relationship between development pattern, travel demand and vehicle capability is the basis for what we called the Local Use Vehicle (LUV) Demonstration Project.

\section{LUV Program Overview}

The first phase of the Local Use Vehicle Demonstration Program began May 1, 2010 and ran for 18 months ending October 31, 2011. Through a funding extension from the AQMD the SBCCOG will continue this program till the end of 2012.

For marketing reasons, the term Local Use Vehicle (LUV) was adopted for the demonstration project in place of the more accurate NEV. These vehicles are also sometimes referred to as slow-speed (SSEV) due to their 25 MPH limit. Technically, LUVs would also include Segways, electric mopeds, and others now in prototype such as GM's EN-V. This initial demonstration project focused on assessing the viability of NEVs on typical suburban streets in today's development pattern.

Through a partnership with Enterprise Rent A Car (ERAC), the SBCCOG leased 5 vehicles:

- Vantage Crewcab

- Columbia Summit

- Wheego Whip (2)

- GEM e4

Subsequently, a GEM dealer loaned the SBCCOG a second e 4 and the City of Santa Monica loaned the SBCCOG a Miles Sedan.

A removable decal with the project logo for easy identification and a GPS unit for tracking usage were installed on each vehicle. Each vehicle was insured by the SBCCOG.

The plan was to loan each vehicle for 6 months to a selected household for unlimited use with no cost to participants other than for battery charging. The 18-month demonstration period would therefore allow 3 rotations so that in the end the project would include 15-18 households.

The initial recruitment was conducted at a street fair in the Riviera Village neighborhood center in south Redondo Beach/west Torrance. Without further recruitment efforts - the vehicles themselves with their project logo served as a rolling advertisement - there are now over 200 interested households on a waiting list.

Because demonstration participants fall into a driving routine within a few weeks that remains 
relatively constant; and in light of the long waiting list, the trial period per household has been reduced to between two and thee months. The data presented below represent the experience of 29 households. The demonstration also included four commercial test cases using a LUV CrewCab.

Most of the participants to date live within the beach cities including Redondo Beach, Manhattan Beach, and portions of Torrance. Participant concentrations have focused around the Rivera Village and a portion of the Artesia corridor allowing the SBCCOG to build off a detailed study of these areas conducted through the South Bay Transportation Performance Study (the 5 year research project that produced the SSBS).

Riviera Village was originally targeted because of its "high capture rate" (percentage of all trips originating in the neighborhood that were captured by the local center). In other words, the SBCCOG initially focused the LUV demonstration in the area where SBCCOG research found a relatively high proportion of very local travel. After focusing on a neighborhood center (Riviera Village) the study was expanded to target the Artesia corridor allowing the SBCCOG to compare the capture rates of center style developments to commercial strip development patterns to determine if there is a difference in vehicle usage.

The usage analysis integrates the following four sources of data:

- GPS on each vehicle, which provides comprehensive tracking of distances, routes, destination locations, time of day, and speeds.

- Hand held GPS units - added in the sixth month - which provide total household VMT for one-week periods.

- Driver logs supplemented by individual interviews that help identify functional destinations from destination locations.

- Focus groups with drivers of each rotational group.

\section{Suburban NEV Usage}

Both the objective data and the personal testimonials reflect a high level of driver acceptance of the NEVs under current conditions.

Based on average monthly GPS data from 29 test placements, each vehicle was driven between 16 and 114 miles per week. The average over all households was 44 miles per week.

Twenty-two of the twenty-eight households also generated trip data from the hand-held GPS units. In those 22 households, the NEV usage ranged between $6 \%$ and $40 \%$ of total household VMT (excluding an outlier where a single resident household used the NEV for $98 \%$ of their total VMT. The average portion of total household VMT driven in NEVs across all households was $22 \%$. Looking just at the driving patterns of the primary driver for each NEV, there is usage range from $14 \%-92 \%$ that averages at $46 \%$.

Looking at variations among groups of drivers one can see a more nuanced picture of how different demographic groups drive. It appears that households with primary drivers between the ages

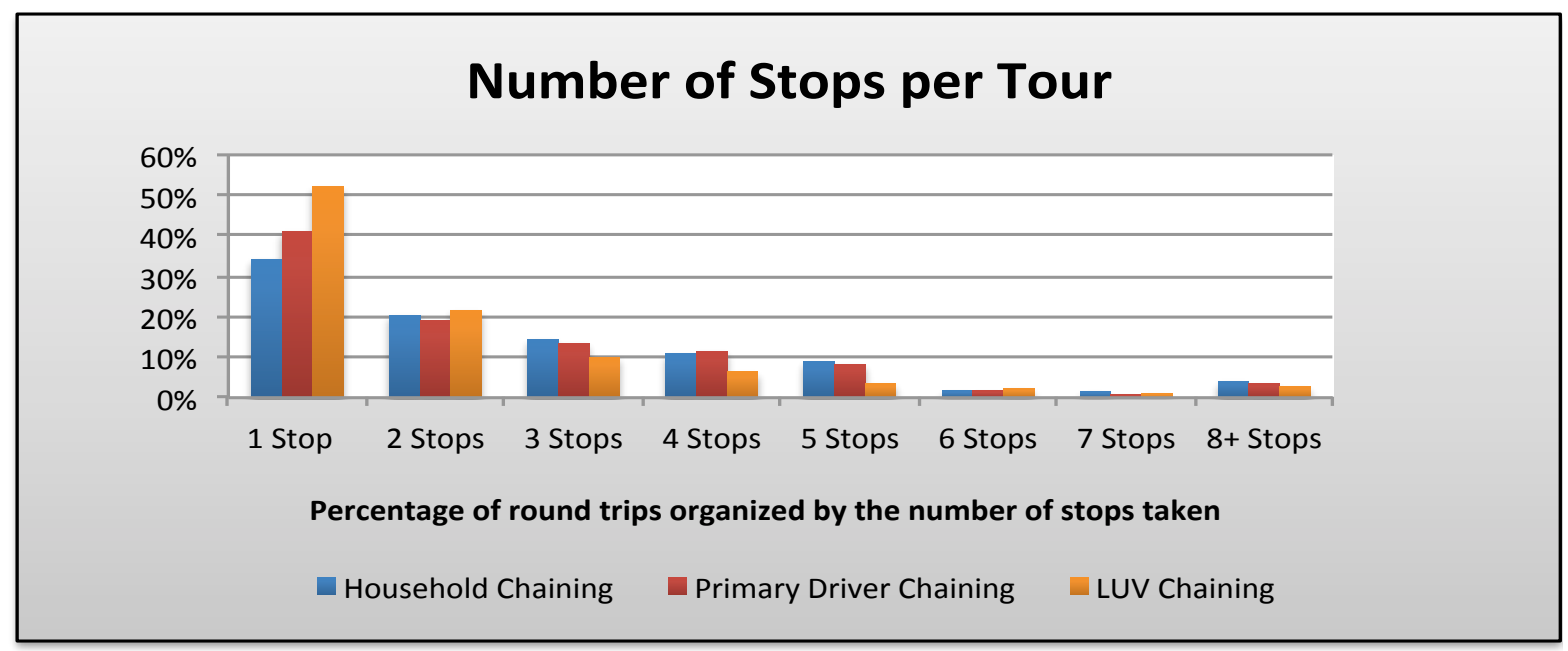

Figure1: Trip Chaining Patterns 


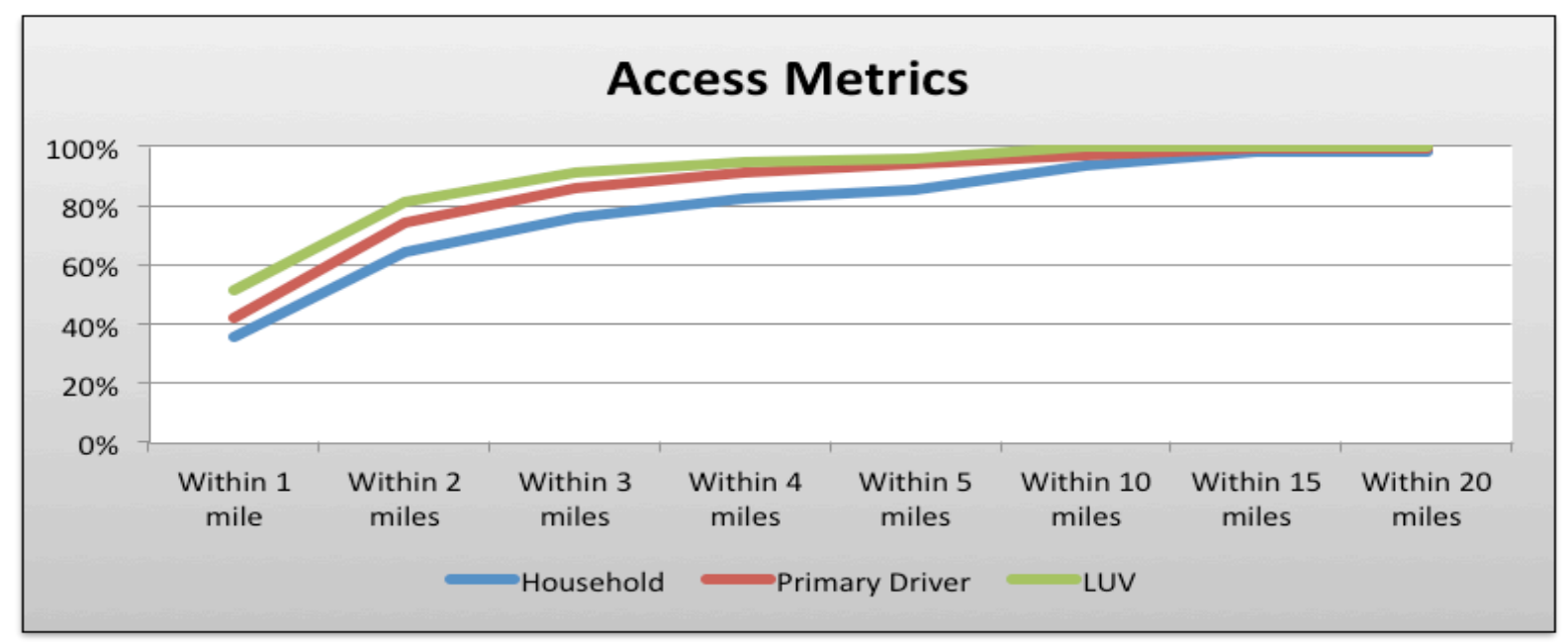

Figure 2: Access Metrics

Percentage of trips taken by distance category

of 45 and 54 use the NEVs more than any other age group.

Because NEVs are not used for long distance trips the NEV usage appears strongest when looking at usage by round trip rather than by mile driven. The NEVs were used for an average of $41 \%$ of trips taken by all household drivers or $72 \%$ of the primary drivers trips. The particular characteristics of these vehicles appear to excel in cold start situations. An average $43 \%$ of all household would be 'cold starts' were prevented by driving a NEV while an average of $90 \%$ of the primary drivers would be 'cold starts' were prevented with the introduction of a NEV.

Figure 1 shows the average number of destinations or 'stops' in a round trip. This chart shows LUVs being driven more frequently when only a single destination is accessed - this is likely to contribute to the cold start elimination figures as chained trips have fewer cold starts than single destination trips.

While NEV drivers drove up to 28 miles in a round-trip, most NEV round trips averaged 4.3 miles with a wide variation among households ranging from an average of 1 - 11 miles per round trip. The trip leg or segment averaged 1.18 miles. Functionally, NEVs have been used most frequently for shopping, errands, going to entertainment venues, and driving to work.

Distances driven in a NEV follow a similar plotted trajectory, as most trips-taken are short distance and are accessible via an NEV. Fifty percent of trips taken in an NEV were less than 1 mile from home, $90 \%$ were less than 3 miles from home, and $96 \%$ were less than 5 miles from home.

Charging was not a barrier for our participants. All charged at home without electric service upgrades or special charging units. About 2/3 of the LUV participants parked and charged in their garages (some that had to get cleaned out to make room for the vehicle). About $1 / 3$ of participating households parked in front of their garage and used an outdoor outlet to charge. There was only one driver from a multi-family residence and that participant ran an adapter from an overhead light fixture in his parking spot.

Two of the drivers reported that they relied so heavily on their NEVs, that when a trip required their regular gasoline-fueled vehicle, the car battery had gone dead from lack of use. Others reported that driving a NEV helped them become more familiar with their own neighborhoods. $80 \%$ of survey respondents indicated that they are more likely to purchase a NEV because of their experience with the demonstration program while $15 \%$ of respondents are less likely to purchase.

In summary: Destinations were usually less than 3 radial miles from home, about 1 driving-mile apart; round-trips averaged about 4 miles in total length due to trip chaining. While the NEV VMT varied greatly by individual and by demographic characteristics, the average percentage of all household travel (VMT) taken in an NEV was 
consistently $22 \%$ across all groups. In terms of vehicle trips, the NEVs mode share averaged $41 \%$ of the round trips.

\section{Environmental Impact}

An interesting result is that NEVs have a more pronounced impact on criteria pollutants than on GHG emissions. The reason is that high volumes of criteria pollutants are emitted when a gasoline engine is first started (a "cold start") and before the catalytic converter reaches a sufficient temperature to eliminate emissions from incomplete combustion. Longer trips tend to produce the majority of GHG emissions and NEVs are ill suited to long trips. Additionally longer trips tend to have higher levels of trip chaining, with fewer cold starts per destination visited than shorter un-chained trips. Because NEVs are primarily driven short distances, driving an NEV heavily mitigates the high levels of cold starts associated with short trip distances.

\begin{tabular}{|l|l|}
\hline \multicolumn{2}{|c|}{$\begin{array}{c}\text { Average Household Emission Reductions } \\
\text { From One NEV }\end{array}$} \\
\hline Hydrocarbons & $28 \%$ \\
\hline Carbon Monoxide & $25 \%$ \\
\hline Nitrogen Oxides & $25 \%$ \\
\hline Particulate Matter 10 & $24 \%$ \\
\hline Particulate Matter 2.5 & $24 \%$ \\
\hline Sulfur Oxides & $23 \%$ \\
\hline Carbon Dioxide & $18 \%$ \\
\hline Methane & $25 \%$ \\
\hline Nitrogen Dioxide & $25 \%$ \\
\hline
\end{tabular}

Table1: Emission Reduction from One NEV

Participating households have reduced their personal transportation related Carbon Dioxide emissions by an average $18 \%^{1}$ or $38 \%$ of the primary drivers $\mathrm{CO} 2$ emissions. Average participating households criteria air pollutants were reduced by $23-28 \%$ depending on the pollutant being measured. Criteria pollutant reductions for the primary driver of the NEV averaged $49 \%$ - 58\% depending on the pollutant being measured.

The emission reduction analysis is based on

1 This statistic reduces to $15 \%$ CO2 reductions when single occupant households are removed from the statistical reporting
- Theoretical power plant emissions per NEV mile driven

- Theoretical trip emissions for each NEV trip taken (if driven in a gas powered vehicle with the emissions of an average passenger vehicle in LA County)

- Theoretical trip emissions for the total household travel demand

Theoretical emission reductions are calculated for each trip taken based on emission factors for cold starts, warm starts, and hot running emissions. These emission factors are based on modeled emission outputs from CARBs EMFAC 2007 emission modeling software for LA County's passenger vehicle fleet. The NEV emission reductions were calculated by subtracting theoretical power plant emissions (calculated on a per mile traveled basis) from the theoretical emissions calculated for each trip taken. Household emission reductions are calculated by dividing the NEV emission reductions by the theoretical emissions of the total household travel demand.

\begin{tabular}{|l|r|}
\hline \multicolumn{2}{|c|}{$\begin{array}{c}\text { Hypothetical Annual Projected Emission } \\
\text { Reductions (Tons) from 150,000 NEVs }\end{array}$} \\
\hline Hydrocarbons & 150.0 \\
\hline Carbon Monoxide & $2,125.3$ \\
\hline Nitrogen Oxides & 201.1 \\
\hline Particulate Matter 10 & 9.7 \\
\hline Particulate Matter $\mathbf{2 . 5}$ & 9.1 \\
\hline Sulfur Oxides & 2.0 \\
\hline Carbon Dioxide & $182,017.9$ \\
\hline Methane & 25.4 \\
\hline Nitrogen Dioxide & 8.3 \\
\hline
\end{tabular}

Table2: Projected Emission Reductions for 150,000

Environmental and Economic Projections: As a near-term target (target date to be determined in discussion with South Bay cities), the SBCCOG hopes to replace approximately $60 \%$ of the second and third vehicles in the South Bay with NEVs and other BEVs. Of the 591,250 passenger vehicles in the South Bay 243,800 of them are second and third vehicles ${ }^{2}$. At $60 \%$ of the secondary fleet, they would replace about 150,000 gas-powered cars. Since there are multiple NEV options

2 Based on data supplied by SCAG 
available in the market today, this process need not wait for new product introduction to begin.

Replacing 150,000 gasoline-powered autos would save over 17 million gallons of gas per year. The carbon emission offsets would be the equivalent of planting over four million trees each year. ${ }^{3}$

Switching 150,000 gas-powered cars to electricpowered cars would save South Bay residents $\$ 67$ million per year. ${ }^{4}$ This would be approximately $\$ 450$ of savings per year for each participating household. This additional \$67 million of disposable income each year would constitute a significant stimulus to the local economy.

\section{Market Barriers}

\subsection{Vehicle speed}

The most significant issue encountered was on main arterials where the posted speed limit may be $35 \mathrm{MPH}$ but the traffic flows closer to 40 or $45 \mathrm{MPH}$ during many periods. NEV drivers experienced discomfort holding up traffic. In response, we added a sticker to the back of each vehicle that said "Local Use Vehicle -- Speed 25 MPH MAX." NEV drivers reported that other drivers became more courteous once the stickers were added.

While those stickers provided drivers with some peace of mind, the practical solution was to avoid arterials where fast traffic might be encountered. This required route planning and experimentation. The project equipped each vehicle with a map showing streets legal for NEVs but in practice drivers tended to apply trial and error in developing preferred routes. In most cases, a parallel street could be found that carried

\footnotetext{
${ }^{3}$ Equivalents are based on the average of NEV test users and EPA GHG Equivalencies Calculator http://www.epa.gov/cleanenergy/energyresources/calculator.html

${ }^{4}$ Assuming \$5/gallon gas, and \$0.06/mile electric car rates as promised by SCE and as measured with energy monitoring devices attached to charging equipment
}

much lower traffic volumes where a slow speed vehicle does not impede other drivers.

Speed can also affect travel time; however our drivers did not mention time as a problem, most likely because of the short trip lengths. Given the limitations on potential driving routes, the SBCCOG thought it would be interesting to see if the available routes proved to be significantly more circuitous than usual. On average, NEVs appear to drive $11 \%$ farther per radial mile traveled due to route restrictions. Between increased driving distance and reduced speed of travel, it appears that NEV travel time is roughly $30 \%$ slower than other vehicles when there is no traffic. During congestion periods LUV drivers report that they are able to get to destinations faster than usual because they are using alternative routes.

\subsection{Neighborhood islands}

There are neighborhoods that are difficult to enter and exit because they are bounded by streets with posted speed limits of $40 \mathrm{MPH}$ and faster. For those cases, the remedy will require establishing separate Class 2 lanes with special striping and signage (which could also accommodate bicycles). A more complex alternative would be the development of a $35 \mathrm{MPH}$ NEV (referred to as Medium Speed Electric Vehicle or MSEV) which would require manufacturers to make improvements to the slow speed NEV models (i.e., electronics, batteries, possibly air bags) and for the National Highway Traffic Safety Administration (NHTSA), which regulates vehicle safety equipment, to approve a medium speed vehicle category.

\subsection{Safety}

No injuries have been sustained in the demonstration program and none of the LUV participants mentioned safety as an issue -- but outsiders often do. The exception was that the Beach Cities Health District had agreed to allow case workers to use two NEVs for trips to the homes of clients, but dropped out of the demonstration following a story in the Los Angeles Times about two NEVs that had essentially failed a crash test used for full speed autos - neither of the 
vehicles tested was in the demonstration fleet. The withdrawal was based on the perception of risk by the Board of Directors of a large nonprofit which suggests participation by such organizations will need to trail rather than lead.

That is not to avoid the reality that NEVs are generally not as crash-ready as full speed vehicles. Risk is minimized by driving them in slow speed environments as has been done in this Demonstration Project.

The flip side of driver safety is the potential threats that motorized vehicles pose to pedestrians, cyclists and other motorists. According to the nation center for Safe Routes to Schools, $12 \%$ of all trips in the SCAG region are done via walking and/or bicycling, yet $25 \%$ of all roadway injuries and fatalities in the region involve pedestrians and bicyclists. Because $\mathrm{NEVs}$ are smaller, lighter, and slower than typical motor vehicles their use will make walking and cycling safer.

\subsection{Terrain}

The South Bay has 3 distinct districts: beach, basin, and Palos Verdes Peninsula (PVP). While the first two are relatively flat, the PVP is quite hilly. The NEVs in this demonstration program were occasionally driven on the PVP, but the hills slowed their speed and tended to drain their batteries. The more powerful BEVs are better candidates for residents of the PVP cities. However, some small market niche for NEVs probably exists as a second vehicle for those living adjacent to one of the commercial centers on the PVP (there are no commercial strips).

\subsection{Range anxiety}

The resistance to driving a BEV for fear of getting stranded with a dead battery is often mentioned as a potential barrier. This has not emerged as a factor so far. Part of the reason is that NEVs are specifically used for local travel. With trips of 3 miles or less and a range of $20+$ miles, home charging is more than adequate for most households.
The exception was in the households with the heaviest use in which drivers took multiple trips in one day. The battery would run down by the end of the day forcing the driver to take the gasolinefueled car sitting in the garage. Even in those cases, elaborate charge port infrastructure (CPI) would not have helped since the NEV was not stopped anywhere for extended lengths of time.

So far there have been two cases of drivers getting stranded. One involved a trip onto the PVP where the batteries wore down from the demands of driving hills -- surprising the driver. The other occurred in the first week of the rotation before the driver had the experience of managing the charge levels. In one case the driver simply waited for the battery to recover (regain its equilibrium) and in the other access to a 110 outlet was borrowed from a friendly resident.

\section{Research Questions}

\subsection{Will residents regularly drive NEVs on typical suburban streets without special lanes or signage?}

Most previous experience with NEVs has been limited to private roads (e.g., on school and medical campuses or in gated communities) and on public roads in golf cart communities. Yet they are legal on public streets with speed limits no faster than $35 \mathrm{MPH}$. Would participants be willing to drive them on "untreated" (those without lane striping or special signage) streets in typical suburban settings?

\section{Answer: Yes}

Both the objective data and the personal testimonials reflect a high level of driver acceptance of the NEVs under current conditions.

\subsection{Will NEV usage produce significant environmental and economic benefits?}

Since adoption of NEVs is not proceeding at a rapid pace on its own, some public policy initiatives will be required to reach fleet transition targets in the South Bay. Is the effort worth it in regards to environmental and economic benefits?

Answer: Yes-emphatically 


\subsection{Is large scale deployment of NEVs feasible?}

The SBCCOG's previous research into the interaction between development pattern and transportation choices suggested that off-theshelf NEVs hold great promise for satisfying significant portions of driving needs without major changes in land use policy, transportation policy or significant infrastructure investments. The LUV Demonstration so far validates that promise.

However, NEVs have been available on the market for 13 years (the first GEM was introduced in April, 1998), and consumers have not embraced them in large numbers. Are there too many barriers or can NEVs actually help the family of BEVs replace the second (and third) vehicle in many households?

\section{Answer: Yes -- conditionally}

\section{Overcoming Market Barriers}

It appears that a concerted, coordinated effort by leading public and private stakeholders could overcome existing barriers and stimulate the private market for NEVs (and other range-limited BEVs). Here are the areas identified so far where action would probably make a difference.

\subsection{Vehicle Price}

Prices must come down. While Current prices for NEVs range from $\$ 8,000$ - $\$ 24,000$, focus group and survey responses indicate consumers are generally not willing to pay more than $\$ 11,000$ for an NEV.

The NEV market niche is dominated by small players with very low production runs. This means they cannot subsidize market development like Nissan, Chevrolet, Honda, and Toyota; nor can they capture economies of scale until demand increases.

The need to lower those price points in the long run illustrates the critical importance of Federal tax credits and State vouchers in the immediate future. The public subsidies should be large enough to bring the price to the consumer close to the targets. The California Air Resources Board (CARB) under the Clean Vehicle Rebate program currently offers NEV vouchers of $\$ 900$ per vehicle (which may be phased out due to
State budget constraints) is inadequate to stimulate the market. Substantial environmental benefits could be captured with a temporary subsidy of $\$ 5,000$ per vehicle.

\subsection{Vehicle Quality}

While quality varied considerably between manufacturers, in general, vehicle quality -- from driver amenities to workmanship and materials needs to improve. For example, seats can be difficult to adjust in one model and the motor on/off switch is placed where it can be accidentally tripped in another. Some vehicles tested had plastic body and/or interior components that were broken or needed adjustment within the first year of ownership due to the use of flimsy plastics and low levels of quality control. All of these quality issues would probably improve with full-scale production. Quality was not a significant deterrent to vehicle use by our participants but quality is sited frequently as the main deterrent from purchasing.

While quality is an area for improvement, it is worth noting that quality has dramatically improved from the early "golf cart" days. Some of our demonstration vehicles have a stylish design, radios and air-conditioning. All had doors. Progress in these areas should improve so long as the NEV market niche remains viable.

\subsection{Local Government}

A range of local government incentives should help. For example free parking in public lots (considered to be a very attractive incentive by our participants but difficult to sustain as the number of EVs increase), parking preference, Class 2 lanes on mixed flow streets where necessary to eliminate speed islands, public charge port infrastructure (CPI), and streamlined permits for private charge port infrastructure by households and businesses wanting to install new or upgrade existing electric meters.

Large-scale use of low speed vehicles is dependent on a network of low speed roads that in some neighborhoods are widely available. In others, there are speed islands (neighborhoods that are bounded by streets posted at $40 \mathrm{MPH}$ and faster) or large specialized centers like an industrial park or retail mall that block low speed travel. Complete 
streets plans and policies are one way to eliminate speed islands.

\subsection{Consumer Education}

Consumers must become more aware of their own driving needs in terms of the proximity to destinations that they currently frequent; and be encouraged to use the vehicle type most appropriate to the trip. This will require a substantial public education program. Gasoline price escalation provides a "teachable moment" as drivers seek fuel-cost relief and become more open to alternatives. A fast moving, multichannel campaign could dramatically increase the rate at which the fleet, particularly the secondary vehicles in a household, can transition away from gasoline fuels to BEVs, especially NEVs.

It is also true that almost all policy makers are either unaware of or have prematurely dismissed the potential contributions of NEVs. Some effort should be invested in reaching the technical staff and decision makers at the regional, state and federal levels.

\subsection{NEV Retailing}

NEV retailers should develop a higher profile. Most people have never heard of Wheego, Miles or Columbia. There are no "auto-rows "which feature competing brands in close proximity to one another. With so many auto dealerships closing during the recession, it should be possible for local governments using state or federal economic development grants in partnership with vehicle manufacturers to re-purpose one such vacant car lot on a demonstration basis.

\subsection{MSEV}

It appears that a medium speed class of NEV capable of 35MPH (i.e., a medium speed electric vehicle or MSEV) would help speed the transition away from gasoline-fueled autos. While most of our drivers enjoyed driving at low speeds, some NEV drivers stated that they would not purchase a LUV unless it was capable of driving at $35 \mathrm{mph}$. An MSEV alternative would reduce the need for Class 2 lanes.

The National Highway Transportation Safety Administration (NHTSA) of the US DOT does not recognize a medium speed vehicle category. Some states such as Wyoming and Hawaii have defied federal regulations by passing MSEV legislation, but California is not one of them. However, some structural changes to the existing slow speed NEV would also be required, so policy change would necessitate a collaborative effort between manufacturers and government regulators.

Because of the difficulty of developing an MSEV category, some research into consumer interest should be conducted as soon as possible. See the discussion in Next Steps for the SBCCOG (below).

\subsection{The Role of NEVs in the BEVs Marketplace}

One of the questions that can only be answered in the marketplace is whether the $25 \mathrm{MPH}$ NEV will compete with the freeway speed BEVs. The five factors identified above would contribute to consumer acceptance, especially price reductions. Once performance needs are better understood, it would seem more likely for consumers to choose the lowest-cost vehicle that meets their travel needs.

Active government intervention in the market place is justified since NEVs serve the broad public interest more effectively than other vehicle options. One reason is that as a vehicle class they are smaller than the more powerful vehicles, including the full-speed BEVs. Size translates into less street congestion and less space for parking in order to accommodate a given volume of vehicles. This also means that parking requirements for new residential and commercial developments can be reduced, thereby lowering construction costs and potentially making NODs more feasible.

NEV drivers typically avoid the high volume, main arterials. This diversion of traffic will also contribute to congestion relief on the high volume roadways.

Another positive attribute of NEVs is that the charging requirements can be less than that required by larger electric vehicles. For example, the Nissan Leaf, a freeway speed BEV with a 100mile range, has a $24 \mathrm{kWh}$ battery capacity. In contrast, the Miles has a $10 \mathrm{kWh}$ battery capacity with a range of 30-40 miles when the battery is new (20-30 on an old battery). A Level 1 charging station (110/120 volts) currently found in every home and most garages is adequate for such small 
battery capacity; whereas the Leaf would justify a Level 2 (220/240 volt) charger which could trigger upgrades including a new circuit, new electric meter, in addition to a separate charging station. The simplicity that comes with low-level technology has its benefits.

Finally, low price, if it can be achieved through economies of scale, would hasten the transition away from gasoline-fueled autos because replacement would occur simultaneously at multiple price points, especially at the low end.

\section{Next Steps for the SBCCOG}

\subsection{Expand the current Demonstration Project}

The SBCCOG has developed the organizational capacity and technical infrastructure for testing and evaluating a range of alternative fuel vehicles. The most cost-effective next step would be to build on these new capabilities and expand the research and demonstration in several dimensions including a demonstration of full speed BEVs.

\subsection{Develop a South Bay Charge Port Infrastructure (CPI) Plan}

BEVs must be plugged in to the electric grid frequently. Home based charging will serve most drivers, just as it has in the demonstration program. However, multi-family residences may not be properly equipped. Barriers to multifamily EVSE infrastructure deployment pose a significant social equity issue. Eventually a network of charge stations at prominent destinations such as employment centers, onstreet parking spaces or public parking lots will be needed. The SBCCOG is currently developing a sub-regional CPI deployment plan.

\subsection{Develop a public education program}

A multi-channel public education program for alerting consumers to the viable electric vehicle options needs to be created. No funding sources for the public education initiative have been identified. At the least the SBCCOG's Environmental Services Center will become a clearinghouse for EV manufacturers' technical and promotional materials.

\subsection{Introduce a car sharing service to the South Bay}

Easy access to a low cost long-range vehicle should help some households replace one or more of their vehicles with BEVs. Preliminary discussions have been held with ERAC about introducing car sharing to the South Bay.

\subsection{Demonstrate a complete streets plan that will eliminate speed islands in a participating city.}

NEVs cannot be legally driven out of those neighborhoods that are speed islands. This limitation effectively constrains the mode options for residents of those neighborhoods. Many speed islands have been identified through this project and the SBCCOG intends to work with local cities to eliminate some speed islands through a demonstration of complete streets planning and creation of Class 2 lanes. The SBCCOG is collaborating with the South Bay Bicycle Coalition in the hopes that some of the proposed Class 2 bicycle lanes will be implemented as "combo lanes" that will accommodate NEVs as well as bicycles.

\subsection{South Bay Mobility Portal}

The SBCCOG is currently seeking funding to develop a Mobility Portal use to inform trip planning, vehicle purchase planning, as well as serving as educational tool and social network forum for local green car drivers. 


\section{Authors}

\section{Wally Siembab}

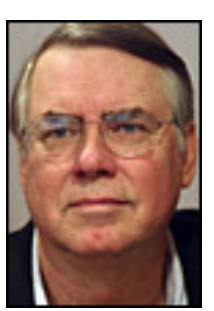

Wally Siembab is the research director for the South Bay Cities Council of Governments. Wally was trained in telecommunications at UCLA and has directed SBCCOG research efforts primarily focused on land use optimization for mature suburbs like the South Bay of Los Angeles.

\section{David Magarian}

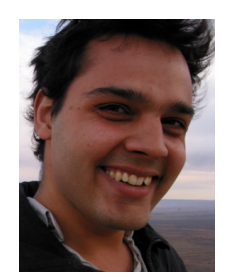

David Magarian manages the Local Use Vehicle Demonstration Program along with other EV and Energy Efficiency programs for the South Bay Cities Council of Governments (SBCCOG). David's background is in green building and urban planning. 